\title{
Eficácia adaptativa e indicadores genéricos de mudança em psicoterapia: um estudo exploratório
}

\author{
Adaptive efficacy and generic indicators of change in psychotherapy: \\ An exploratory study
}

\author{
Giovanna Corte HONDA ${ }^{1}$ \\ Elisa Médici Pizão YOSHIDA² \\ Mariane KRAUSE ${ }^{3}$ \\ Guillermo de la PARRA ${ }^{3}$
}

\begin{abstract}
Resumo
Esta pesquisa exploratória objetivou avaliar a relação entre evolução dos indicadores genéricos de mudança e qualidade da eficácia adaptativa de uma paciente adulta atendida em psicoterapia breve psicodinâmica. Os vídeos e transcrições das 11 sessões deste processo foram analisados com os Indicadores Genéricos de Mudança e com a Escala Diagnóstica Adaptativa Operacionalizada Redefinida. Os resultados demonstraram evolução dos indicadores de mudança e progresso nas respostas adaptativas. Se por um lado respostas mais adequadas sugeriram mudanças na vida da paciente, por outro, essas melhoras puderam ser captadas pelos Indicadores Genéricos de Mudança. As duas medidas usadas em conjunto podem auxiliar o terapeuta na avaliação de progresso do paciente e na compreensão dos fatores que ocasionam ou que limitam a mudança. Sugere-se a realização de pesquisas que compreendam maior número de casos, contem com processos considerados mal sucedidos e envolvam abandono por parte do paciente.
\end{abstract}

Palavras-chave: Estudos de caso; Intervenção psicológica; Mudança (psicologia); Processos psicoterapêuticos.

\begin{abstract}
This exploratory study aimed to evaluate the relationship between the progress on generic indicators of change and the quality of adaptive efficacy in an adult patient who underwent brief psychodynamic psychotherapy. The videos and transcripts of 11 therapy sessions were analyzed using the "Indicadores Genéricos de Cambio" (Generic Change Indicators) and the "Escala Diagnóstica Adaptativa Operacionalizada Redefinida" (Operational Adaptive Diagnostic Scale Redefined). The results pointed to the progress on the indicators of change and progress in adaptive responses. More adequate responses suggested changes in the patient's life, and these improvements were detected by the Generic

${ }^{1}$ Universidade Nove de Julho, Departamento de Saúde III. Av. Dr. Adolpho Pinto, 109, Barra Funda, 01156-050, São Paulo, SP, Brasil. Correspondência para/Correspondence to: G.C. HONDA. E-mail: <giovanna151@yahoo.com.br>.

2 Pontifícia Universidade Católica de Campinas, Centro de Ciências da Vida, Programa de Pós-Graduação em Psicologia como Profissão e Ciência. Campinas, SP, Brasil.

${ }^{3}$ Pontifícia Universidad Católica de Chile, Escuela de Psicología, Departamento de Psiquiatría. San Joaquin, Santiago de Chile, Chile. Artigo baseado na tese de G.C. HONDA, intitulada "Indicadores Genéricos de Mudança em Psicoterapia e Eficácia Adaptativa". Pontifícia Universidade Católica de Campinas, 2014

512 Apoio: Coordenação de Aperfeiçoamento de Pessoal de Nível Superior (Processo no 19190-12-3).
\end{abstract}


Change Indicators. Used together, these two measures may assist the therapist in evaluating patient's progress and understanding the factors that cause or limit change. Further studies with a larger number of psychotherapy processes and which take into account unsuccessful processes and patient abandonment are recommended.

Keywords: Case studies; Psychological intervention; Change (psychology); Psychotherapeutic processes.

A busca por psicoterapia se dá usualmente quando o indivíduo está enfrentando alguma dificuldade ou sentimento que o tornam insatisfeito consigo próprio e/ou com outros de seu ambiente. Tratam-se de situações para as quais não encontram soluções satisfatórias, além de implicarem algum tipo de conflito interno e/ou externo. O propósito seria o de encontrar soluções consideradas mais adequadas, no sentido de solucionar o problema, obter mais prazer e resolver os conflitos existentes, condições que, de acordo com Simon (1997), definem a eficácia da adaptação.

Nesse sentido, é possível afirmar que processos de psicoterapia têm como principal foco ajudar as pessoas a se adaptarem de forma mais eficaz e obter alívio para seu sofrimento psíquico. Essa asserção se aplica a todas as psicoterapias, independentemente da orientação teórica do terapeuta ou da modalidade de atendimento (individual, casal, familiar ou grupal) (Honda \& Yoshida, 2013). Por outro lado, em todas as psicoterapias é possível identificar fatores relevantes para o progresso do paciente, os quais são reconhecidos como sendo comuns (Krause et al., 2006; Krause \& Altimir, 2016; Wampold, 2015). A título de exemplo, pode-se citar a aliança terapêutica, ou seja, a qualidade do relacionamento entre o terapeuta e o paciente e que decorre do grau de confiança, intimidade e segurança compartilhada por ambos ao longo do processo (Barber et al., 2014; Wiseman \& Tishby, 2014).

Outros fatores comuns seriam a expectativa do paciente quanto ao auxílio que receberá na psicoterapia (expectativa diz respeito ao sentimento de esperança e de confiança em relação ao tratamento, ao psicoterapeuta e aos benefícios que poderá obter) (Barber et al., 2014; Constantino, Vîslă, Ogrodniczuk, Coyne, \& Söchting, 2016; Wampold, 2015); a motivação do paciente para enfrentar seus problemas e iniciar o tratamento; e a participação ativa no processo psicoterápico, que demonstra a forma como o paciente encara suas dificuldades: encarando os problemas ou tentando evitá-los (Jung, Serralta, Nunes, \& Eizirik, 2015).

Considerando que fatores comuns contribuem para os resultados de todas as psicoterapias, podese falar, em acordo com Krause et al. (2006), em mudança terapêutica genérica, a qual pode ser didaticamente pensada segundo duas dimensões: conteúdo e evolução. A dimensão conteúdo refere-se à perspectiva que o paciente tem de si mesmo, suas dificuldades, sintomas e a relação que estabelece entre eles. Ou seja, "... às transformações da representação de si mesmo e das relações com o ambiente" $^{4}$ (Krause et al., 2006, p.318, tradução nossa). Quanto à dimensão evolução, esta supõe que a "... mudança subjetiva se compõe de uma sequência de mudanças sucessivas, na qual as mudanças posteriores se baseiam nas anteriores e as englobam" 5 (Krause et al., 2006, p.318, tradução nossa). Os autores concluem, então, que o que muda em uma psicoterapia é "... a teoria subjetiva, a narrativa interna, que se constrói progressivamente à medida que vão se reunindo os novos significados que se fazem visíveis ao observador através de indicadores de mudança" 6 (Krause et al., 2006, p.320, tradução nossa).

\footnotetext{
${ }^{4}$ Do original: "que dice relación con transformaciones de la representación de sí mismo y de las relaciones con el entorno" (Krause et al., 2006, p.318).

${ }^{5}$ Do original: "cambio subjetivo se compone de una secuencia de cambios sucesivos, en que los cambios posteriores se basan en los anteriores y los engloban" (Krause et al., 2006, p.318).

${ }^{6}$ Do original: "lo que cambia es la teoría subjetiva, la narrativa interna, que se construye progresivamente a medida que se van ensamblando los nuevos significados, que se hacen visibles al observador a través de los indicadores de cambio" (Krause et al., 2006, p.320).
} 
Esses indicadores podem ser apreendidos por meio de estudos de eventos ou episódios, através dos quais é possível observar um momento de mudança e explicar as variedades nos resultados dos tratamentos psicoterápicos. Entende-se por momento de mudança o ponto mais "culminante" e significativo tratado pelo paciente e que pode ser observado nas sessões ou em situações extrassessões por ele narradas. Já um episódio de mudança engloba o segmento da sessão que antecede o momento de mudança, isso significa que é anterior ao ponto culminante e que leva à mudança, paciente e psicoterapeuta já devem estar falando sobre o tema em questão (Krause et al., 2006; Krause, Altimir, Pérez, \& de la Parra, 2014).

Com o propósito de viabilizar a observação e avaliação do que ocorre dentro e ao longo das sessões psicoterapêuticas, foi desenvolvido um instrumento conhecido como Indicadores Genéricos de Mudança em Psicoterapia (IGM) (Krause et al., 2006, 2007). Está fundamentado numa sequência ideal de mudanças que pode ser observada empiricamente durante sessões de psicoterapia. Mais especificamente, reúnem uma hierarquia de indicadores genéricos os quais sinalizam se alguma mudança está ocorrendo no processo psicoterápico e se se dá no sentido de progresso. Parte-se do pressuposto de que o início da mudança pode ocorrer antes da terapia, mas se evidencia com o pedido de ajuda a um profissional.

Um primeiro indicador de mudança nesse estágio envolve a manifestação de dúvidas relacionadas à maneira de viver a própria vida e de enfrentar as dificuldades inerentes a ela (Krause et al., 2007). Esse fato pode demonstrar que o paciente aceita a existência de um problema, se depara com os próprios limites e, por isso, reconhece a necessidade de auxílio. O tratamento também depende da anuência do paciente em participar ativamente do processo, bem como de se responsabilizar pelas eventualidades que ocorrem em sua vida (Krause et al., 2006).

Depois de essas primeiras mudanças serem

514 alcançadas, espera-se o surgimento de sentimentos de competência, além de novas representações afetivas e cognitivas que ajudam o paciente a obter insights e adquirir nova compreensão acerca de suas dificuldades. Para esse processo, contribuem as intervenções e atitudes do terapeuta em relação às dificuldades do paciente, assim como os eventos de seu ambiente. A ocorrência dos indicadores aponta para novas atitudes e comportamentos da pessoa em relação a si mesma e ao contexto em que vive (Krause et al., 2007). Em consequência, ocorrem mudanças nas teorias subjetivas do paciente, ou seja, passa a elaborar novos modos de interpretar e de explicar a visão que tem de si mesmo e do entorno.

Os Indicadores Genéricos de Mudança aplicam-se para situações intra e extrassessão e, por isso, apresentam uma vantagem sobre instrumentos que levam em consideração apenas o que acontece dentro da sessão. Além disso, pode ser útil para monitorar o progresso em psicoterapia e fornecer uma visão hierarquizada sobre como ocorre a mudança nesse contexto (Honda \& Yoshida, 2013).

É possível mencionar alguns estudos no cenário atual das pesquisas que usam o IGM (Altimir et al., 2010; Fernández, Pérez, Gloger, \& Krause, 2015; Krause et al., 2014). O trabalho de Altimir et al. (2010) buscou identificar e analisar mudanças a partir de uma perspectiva tripla: avaliação do paciente, do terapeuta e de observadores externos às sessões. Para tanto, foram estudados 27 processos psicoterápicos de diferentes abordagens e modalidades terapêuticas. As mudanças percebidas tanto pelo paciente quanto pelo terapeuta foram identificadas a partir de entrevista semiestruturada, no follow up de dois a quatro meses após o término da terapia, e foram categorizadas de acordo com os IGM. Quanto aos observadores, estes analisaram em pequenos grupos as sessões de psicoterapia ao vivo (em uma sala de espelho) e buscaram pela presença de IGM. Os autores verificaram que paciente, terapeuta e observadores externos concordaram ser a manifestação de novos comportamentos e emoções o conteúdo mais representativo de mudança em todas as psicoterapias. Em terapias exitosas, os pacientes reportaram indicadores de 
maior nível hierárquico, ou seja, para o paciente as mudanças alcançadas são mais perceptíveis do que para os terapeutas e observadores.

Krause et al. (2014) analisaram a relação entre mudanças ao longo de processos terapêuticos utilizando os IGM com os resultados das psicoterapias em 39 pacientes. O estudo constatou que os resultados das terapias dependeram das mudanças ocorridas durante o processo. Aquelas consideradas exitosas contaram com a presença de indicadores de maior nível hierárquico. Nesses casos, houve uma associação significativa entre a presença de indicadores de menor nível hierárquico e a conquista de indicadores mais elevados. Essa relação, no entanto, não foi observada em terapias consideradas sem êxito, as quais alcançaram maior número de indicadores iniciais.

Já o estudo realizado por Fernández et al. (2015) buscou avaliar a evolução dos IGM em fases iniciais de psicoterapias de adolescentes e sua relação com a adesão ao tratamento. Observaram maior presença de indicadores de níveis hierárquicos menos elevados na primeira sessão, quando comparados com a segunda e terceira sessões. Notaram, ainda, que à medida em que um indicador de nível hierárquico mais elevado é alcançado ao longo do tratamento, a aderência à terapia aumenta, existindo menor possibilidade de desistência durante o processo.

Tomando como base o conceito de hierarquia de indicadores genéricos de mudança (Krause et al., 2006, 2007) e de eficácia adaptativa, nos termos propostos por Simon (1997), Honda e Yoshida (2013) sugerem que eles se complementam e ajudam na compreensão de como se processa a mudança em psicoterapia. As autoras argumentam que as mudanças nas teorias subjetivas do paciente, passíveis de serem acompanhadas por meio dos indicadores, refletem mudanças na qualidade de suas respostas adaptativas e, por conseguinte, em sua eficácia adaptativa. Nesse sentido, sugerem a realização de pesquisas empíricas onde a possível associação entre essas medidas possa ser verificada. Defendem especialmente investigações com delineamento qualitativo, em que processos de casos únicos (Eells,
2007) sejam analisados no sentido de se avançar na compreensão sobre como se processa a mudança em psicoterapia. Essa metodologia, baseada em gravações em vídeo e transcrições das sessões, permite analisar em profundidade o que ocorre a cada momento em um processo terapêutico.

Dessa maneira, a presente pesquisa, de cunho exploratório, avaliou a eficácia adaptativa e os indicadores genéricos de mudança em conjunto. Partiu-se da suposição de que a evolução hierárquica dos indicadores genéricos de mudança é acompanhada de melhora na qualidade adaptativa das respostas do paciente, especialmente no setor da personalidade relacionado ao foco de uma psicoterapia. O objetivo específico foi o de examinar a relação entre evolução dos indicadores genéricos de mudança e a qualidade da eficácia adaptativa de uma paciente atendida em psicoterapia breve de orientação psicodinâmica.

\section{Método}

\section{Participante}

Mulher, 48 anos, com ensino médio completo, desquitada, com três filhos casados. $\mathrm{Na}$ ocasião da terapia estava afastada do serviço por doença - era diabética e utilizava medicação antidepressiva. Foi encaminhada à clínica-escola por seu psiquiatra.

\section{Terapeuta}

Mulher, doutora em Psicologia, com 33 anos de prática clínica.

\section{Juízes}

J1 - Psicólogo, doutorando em Psicologia, membro do grupo de pesquisa "Psicoterapia Breve Psicodinâmica: Avaliação de Mudança e Instrumentos de Medida da Pontifícia Universidade Católica 
de Campinas (PUC-Campinas)"; J2 - Psicóloga, doutoranda em Psicologia, membro do grupo de pesquisa "Psicoterapia Breve Psicodinâmica: Avaliação de Mudança e Instrumentos de Medida da PUC-Campinas"; 13 - Psicóloga, doutoranda em Psicologia, membro do grupo de pesquisa "Psicoterapia Psicodinâmica Breve: Avaliação de Mudança e Instrumentos de Medida da PUCCampinas"; J4 - Psicólogo, doutorando em Psicologia, membro do grupo de pesquisa "Núcleo Milenio Intervención Psicológica y Cambio en Depresion" (PUC-Chile); J5 - Psicóloga, doutora em Psicologia; J6 - Psicóloga, doutora em Psicologia; J7 - Psicólogo, doutor em Psicologia.

\section{Resumo do processo terapêutico}

Paciente procurou a clínica-escola de Psicologia com queixa de insônia e de se sentir prisioneira de suas obrigações. Há cerca de oito meses vinha cuidando do ex-marido, que teve um derrame, e desde então sentia que não conseguia se distrair e nem viver a própria vida. Acreditava que nunca tivera tempo para isso. Diante do conflito existente entre o sentimento de dever para com o ex-marido, por se encontrar incapaz, e o fato de querer viver a vida, o objetivo do processo terapêutico consistiu em solucionar esse conflito, com o intuito de diminuir o sentimento de culpa e a agressão contra si mesma - sobre isso, relatou explicitamente preferir prejudicar-se para que todo mundo pudesse viver em harmonia, no entanto, não se sentia bem com essa assertiva, pois colocava sua saúde em risco.

Fatos importantes na história de vida foram averiguados: paciente era a filha mais velha e desde criança trabalhou em casa e cuidou dos irmãos, pois a mãe era doente. Também sofria castigos físicos do pai, que era alcoólico. Casou-se grávida aos 18 anos com um homem que era alcoólico desde os 11, porém só soube que tinha problemas com bebida quando o primeiro filho nasceu. Depois do nascimento do terceiro filho, começou a trabalhar e a ganhar mais que o marido. Houve brigas durante o casamento e os problemas com o álcool foram se tornando piores. Separou-se, e o agora ex-marido passou a beber ainda mais. Dois anos antes de iniciar a terapia, a paciente mudou-se para a casa da mãe, que se encontrava doente. No ano seguinte, a mãe veio a falecer e a paciente entrou em depressão severa. Meses depois, passou a cuidar do ex-marido que sofreu derrame. Os três filhos, embora descritos pela paciente como exemplares, não a ajudavam o suficiente nos cuidados. Paciente afirmou que gostava das três noras e evitava conflito com elas.

\section{Instrumentos}

Indicadores Genéricos de Mudança (IGM) - É uma lista desenvolvida por Krause et al. $(2006,2007)$ com base em investigações qualitativas prévias. Formada por indicadores de mudanças sucessivas que podem ser empiricamente observados em sessões psicoterapêuticas, sendo eles, em ordem hierárquica:

(1) Aceitação da existência de um problema; (2) Aceitação dos próprios limites e da necessidade de ajuda; (3) Aceitação do psicoterapeuta como sendo um profissional competente; (4) Expressão de esperança ("Remoralização" ou expectativa de ser ajudado ou de superar os problemas); (5) Questionamento de habituais formas de entendimento, comportamentos e emoções (pode indicar reconhecimentos de problemas anteriormente não vistos, autocrítica ou redefinição das expectativas e objetivos terapêuticos); (6) Expressão da necessidade de mudança; (7) Reconhecimento e/ou responsabilidade na participação dos próprios problemas; (8) Descobrimento de novos aspectos de si mesmo; (9) Manifestação de novos comportamentos e/ou emoções; (10) Aparecimento de sentimentos de competência; (11) Aparecimento de novas conexões entre: aspectos do eu (crenças, comportamentos, emoções), aspectos do eu e do ambiente (pessoas ou eventos), aspectos 
do eu e elementos da própria história de vida; (12) Reconceituação dos próprios problemas ou sintomas; (13) Transformação de valores e emoções em relação a si mesmo a aos outros; (14) Formação de novos construtos subjetivos do eu através da interconexão de aspectos pessoais e de aspectos do ambiente, incluindo problemas e sintomas; (15) Enraizamento dos constructos subjetivos na própria história de vida; (16) Compreensão e utilização autônoma de contextos de significados psicológicos; (17) Reconhecimento da ajuda recebida; (18) Diminuição da assimetria entre paciente e psicoterapeuta; (19) Construção da própria história de vida fundamentada em teorias subjetivas do eu e na relação com o ambiente (Krause et al., 2006, p.310; tradução nossa) ${ }^{7}$.

O instrumento é empregado para avaliação de mudança de processos psicoterapêuticos, a partir da análise de sessões transcritas de áudio e vídeo. O avaliador deve identificar momentos de mudança seguindo quatro critérios: (1) Correspondência teórica: a mudança deve vislumbrar o conteúdo de um indicador genérico de mudança; (2) Verificabilidade: a mudança pode ser observada dentro da sessão e, caso seja uma mudança extrassessão, a mesma é mencionada explicitamente durante a psicoterapia; (3) Novidade: o conteúdo de mudança deve ser manifestado pela primeira vez; (4) Consistência: a mudança é consistente com uma comunicação não verbal, que pode ser observada em gravações de vídeo, e posteriormente não é negada em sessão, ao longo da psicoterapia.

Com base nesses quatro critérios e na hierarquia dos IGM, o avaliador identifica e especifica todos os momentos e episódios de mudança. Vale ressaltar que a hierarquia dos IGM foi desenvolvida e validada em dois trabalhos (Arístegui et al., 2004; Krause, 2005). Embora existam índices aplicáveis para a amostra brasileira, pesquisas internacionais mostraram alto grau de acordo intersubjetivo entre juízes treinados nos IGM (Altimir et al., 2010; Fernández et al., 2015; Krause et al., 2014).

Escala Diagnóstica Adaptativa Operacionalizada Redefinida (EDAO-R) (Simon, 1997): avalia a eficácia adaptativa por meio da qualidade das respostas do indivíduo frente às vicissitudes da vida. Essas podem ser classificadas como: adequadas, pouco adequadas ou pouquíssimo adequadas, em função do grau em que resolvam o problema, tragam satisfação e evitem conflito interno ou externo. As respostas são apreciadas segundo dois setores do funcionamento da personalidade: afetivo-relacional (A-R) e produtividade (Pr). Ao setor A-R, atribui-se para adaptação adequada o escore 3; para pouco adequada, escore 2; e 1 para pouquíssimo adequada. Em relação ao setor Pr, as pontuações são respectivamente: 2; 1; e 0,5. Pesquisas voltadas para as qualidades psicométricas de precisão e validade evidenciam que o instrumento possui um alto grau de acordo entre juízes ( $k=0,78$ para a escala total; $k=0,78$ para o setor A-R e $k=1$ para o setor Pr), além de oferecer critérios confiáveis para a indicação e contraindicação de pacientes para psicoterapias breves (Yoshida, 1999).

\footnotetext{
$\boldsymbol{\nabla} \boldsymbol{\nabla} \boldsymbol{V}$

${ }^{7}$ Do original: 1. Aceptación de la existencia de un problema. 2. Aceptación de los propios límites y reconocimiento de la necesidad de ayuda. 3. Aceptación del terapeuta como un profesional competente. 4. Expresión de esperanza ("remoralización" o expectativa de ser ayudado o de superar los problemas). 5. Descongelamiento (o cuestionamiento) de formas de entendimiento, comportamientos y emociones habituales ("fisura"). Puede implicar reconocimiento de problemas antes no vistos, autocrítica y/o redefinición de expectativas y metas terapéuticas. 6. Expresión de la necesidad de cambio. 7. Reconocimiento de la propia participación en los "problemas". 8. Descubrimiento de nuevos aspectos de sí mismo. 9. Manifestación de un comportamiento o de una emoción nuevos. 10. Aparición de sentimientos de competencia. 11. Establecimiento de nuevas conexiones entre: - Aspectos propios (creencias, conductas, emociones, etc.). - Aspectos propios y del entorno (personas o sucesos). - Aspectos propios y elementos biográficos. 12. Reconceptualización de los propios problemas y/o sintomas. 13. Transformación de valoraciones y emociones en relación a sí mismo u otros. 15. Formación de constructos subjetivos respecto de sí mismo a través de la interconexión de aspectos personales y del entorno, incluyendo problemas y sintomas. 16. Enraizamiento de los constructos subjetivos en la propia biografía. 17. Autonomía en cuanto al manejo propio del contexto de significado psicológico. 18. Reconocimiento de la ayuda recibida. 19. Disminución de la asimetría entre paciente y terapeuta (Krause et al., 2006, p.310).
} 
Não existem pontos de corte para as avaliações dos IGM e da eficácia adaptativa. Como se trata de estudo de processo, o progresso do paciente pode ser considerado mudança em psicoterapia. Ressalta-se que essas medidas requerem juízes familiarizados com os respectivos instrumentos, pois estes envolvem conhecimentos teóricos e clínicos, que demandam treino por parte dos avaliadores. O emprego adequado das ferramentas pode trazer informações importantes acerca do paciente e de seu processo de mudança.

\section{Procedimentos}

O processo de 11 sessões contou com gravação em vídeo e transcrição das sessões e foi selecionado dentre outros três processos de psicoterapias breves utilizados em projeto de pesquisa guarda-chuva (Yoshida, 2008) devido ao fato de ter sido considerado clinicamente bem-sucedido. Isto é, não havia mais queixa de insônia, a paciente obteve o apoio de seus filhos nos cuidados de seu ex-marido e fazia planos para vir a se casar novamente. A pesquisa foi realizada com aprovação do Comitê de Ética em Pesquisas com Seres Humanos da PUCCampinas (Parecer $n^{\circ} 177.948 / 2012$ ). A paciente foi contatada para a obtenção de autorização para a utilização dos dados nessa nova investigação e assinou novo Termo de Consentimento Esclarecido.

Primeiramente, as transcrições do processo psicoterápico foram analisadas por meio da EDAO-R (Simon, 1997) por dois juízes independentes, visando a avaliação da configuração adaptativa no início e no final do processo (J1 e J2). Foram analisadas a $1^{\mathrm{a}}$ e $2^{\mathrm{a}}$ sessões (correspondentes à fase inicial) e a $10^{\mathrm{a}}$ e $11^{\mathrm{a}}$ sessões (correspondentes à fase final). Na segunda etapa, para a avaliação da IGM, dois outros juízes (J3 e J4), que receberam treinamento formal com os responsáveis pelo desenvolvimento do instrumento, assistiram aos vídeos do processo psicoterápico completo e identificaram se os episódios continham os critérios de correspondência teórica, verificabilidade, novidade e consistência. De um total de 18 episódios encontrados, houve acordo em 14 deles. Os que não obtiveram acordo foram excluídos para manter maior rigor metodológico.

De posse da localização dos episódios de mudança, os trechos correspondentes foram avaliados por outros dois juízes independentes (J5 e J6) e cegos quanto aos objetivos da pesquisa. Estes avaliaram a qualidade das respostas adaptativas correspondentes ao conteúdo tratado em cada episódio identificado (total de 14 fragmentos de sessões). Para aumentar o rigor metodológico, os episódios de mudança foram passados aos juízes em ordem não cronológica, de forma que não soubessem em que etapa do processo cada um ocorreu. Assim, quando não houve acordo entre eles, um terceiro juiz (J7) foi consultado e, no caso de acordo entre o terceiro juiz e um dos dois primeiros, a avaliação foi aceita. Ressalta-se que a terapeuta não foi treinada na avaliação dos indicadores genéricos de mudança.

\section{Resultados}

A Figura 1 apresenta a avaliação da qualidade da adaptação de cada setor (A-R e Pr) com a EDAO-R e respectivas avaliações da eficácia adaptativa nas fases inicial (1 a e 2a sessões) e final da psicoterapia (10a e 11a sessões). O principal conflito observado no início, que justifica a adaptação pouquíssimo adequada no setor $A-R$, relaciona aos cuidados que a paciente despendia ao ex-marido. Expressou várias vezes o quanto gostaria de viver a própria vida, mas se sentia presa à obrigação de "cuidar do pai de seus filhos" [sic]. A paciente explicou que preferia não pedir ajuda, porque, se o fizesse, estaria atrapalhando a vida dos filhos e das noras. Preferia prejudicar-se para não entrar em atrito com ninguém. Nas sessões finais, no entanto, ela começou a mostrar suas limitações físicas aos filhos que, contrariando suas expectativas, passaram a assumir mais os cuidados com o pai. Nesse estágio, houve melhora do relacionamento dela com os filhos. As respostas que passou a apresentar nesse setor trouxeram-Ihe satisfação e não causavam mais os conflitos até então sofridos. 


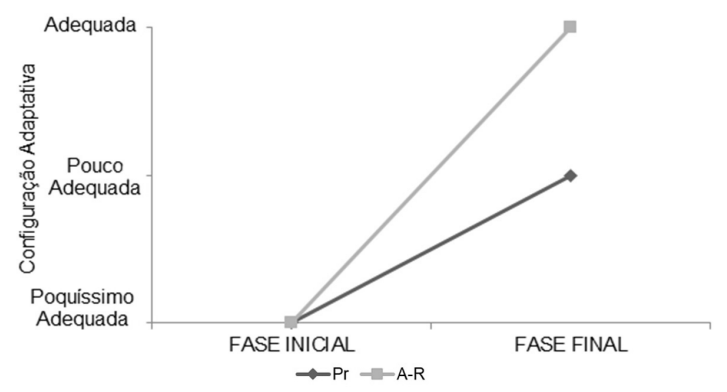

Figura 1. Avaliação da EDAO-R nas fases inicial e final do processo terapêutico.

Nota: A-R: Afetivo-Relacional; Pr: Produtividade.

Em relação ao setor Pr, no início do tratamento a adaptação também foi considerada pouquíssimo adequada. A paciente trabalhava como assistente de cobrança em uma empresa e no momento da terapia estava afastada do emprego por doença (diabetes). Realizava perícias médicas regularmente para ser avaliada quanto à possibilidade de voltar ao serviço e, apesar disso, relatou não querer voltar a trabalhar, pois estava fazendo seu serviço contrariada. Para ela, o ambiente de trabalho era hostil e recebia pouco reconhecimento de seus superiores. Todos esses sinais sugerem a ausência de gratificação no emprego e a presença de conflito (possibilidade de voltar a trabalhar após passar por nova perícia e desejo de não querer que isso acontecesse).

No final dos atendimentos, o setor $\mathrm{Pr}$ apresentou pequena evolução (foi avaliado como pouco adequado). Nesse caso, a paciente relatou que sentia vontade de voltar a trabalhar em outro ambiente e tinha novas perspectivas de trabalho. Ainda não se encontrava satisfeita com sua condição atual, pois o fato de essa pendência ainda não estar resolvida trazia muitas preocupações.

Quanto à identificação dos IGM (Krause et al., 2006), foram encontrados 14 episódios de mudança intra ou extrassessão, distribuídos nas 11 sessões da terapia (Figura 2). A sessão nove foi a única em que nenhum episódio de mudança foi localizado.

Observa-se que houve evolução dos indicadores de mudança desde a primeira até a última sessão, ainda que isso não tenha ocorrido

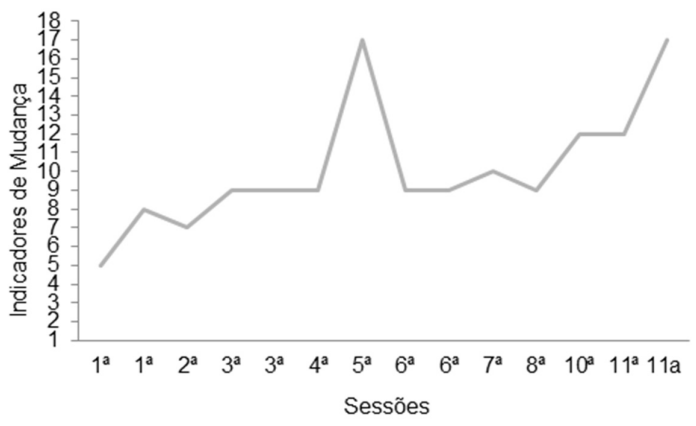

Figura 2. Evolução dos IGM ao longo das sessões de psicoterapia. Nota: IGM: Indicadores Genéricos de Mudança.

de forma linear. As sessões iniciais abarcaram indicadores de menor hierarquia (indicador 5; indicador 7; e indicador 8), enquanto que as finais englobaram indicadores de maior hierarquia (indicador 12; indicador 17).

\section{Discussão}

A comparação das respostas adaptativas da paciente, no início e no final do processo terapêutico sugere que teria se beneficiado com o atendimento, passando a enfrentar suas dificuldades de forma mais eficaz. É possível que a principal mudança tenha se processado no grau de rigidez de alguns traços, como, por exemplo, um abrandamento na convicção demonstrada inicialmente de que deveria poupar seus entes queridos de sofrimentos ou de responsabilidades mesmo que, para tanto, devesse se sacrificar e pôr em risco a própria saúde. Outra mudança observada seria na possibilidade de desfrutar de certa liberdade interior para se permitir fazer coisas que lhe trouxessem prazer, sem que isso implicasse em abandonar tudo e todos.

A par das mudanças na esfera A-R, houve também mudança na forma com que a paciente passou a enfrentar questões relacionadas ao trabalho, ainda que persistisse um nível de conflito, o que caracteriza uma solução pouco adequada nesse setor. É importante ressaltar, todavia, que o foco do atendimento ateve-se a situações de natureza afetivo-relacional e que mudanças no setor da Pr não eram necessariamente esperadas. 
Ainda que a comparação da configuração adaptativa inicial e final sugira que a psicoterapia foi bem-sucedida, é preciso notar que as mudanças foram paulatinas. A análise sessão a sessão dos episódios de mudança, concomitantemente à avaliação da qualidade da adaptação, contribuiu especialmente com elementos para a compreensão de como as mudanças teriam se processado.

Em conformidade com o defendido na literatura, essa evolução provavelmente ocorreu em função da presença de vários fatores, que podem repercutir diretamente nos resultados do tratamento. Esses podem ser representados por variáveis relacionadas ao paciente, ao psicoterapeuta e à qualidade da relação entre ambos. No que diz respeito a essa última variável, percebeu-se que desde o início do tratamento foi estabelecida uma sólida aliança de trabalho entre terapeuta e paciente, o que é apontado por vários autores como um importante elemento para o sucesso de uma terapia (Wiseman \& Tishby, 2014).

Para a participante, alguns dos fatores que podem ter contribuído para sua melhora dizem respeito à sua participação ativa no processo e o nível de consciência em relação ao problema, refletindo em sua motivação para trabalhar as próprias dificuldades. No que concerne a esses elementos, é possível perceber que, apesar de ter sido encaminhada pelo psiquiatra, a paciente tinha consciência de que suas dificuldades estavam influenciando seu estado emocional e sua saúde física, propiciando, provavelmente, a assunção de uma postura ativa para superar suas questões. Além disso, demonstrou contar com recursos internos para enfrentar os problemas, fato verificado pela forma como lidou com os acontecimentos da sua vida no passado (em momentos de dificuldade, se mostrava batalhadora, indo em busca de seus objetivos). Esses dados corroboram a observação de vários autores de que o envolvimento ativo do paciente durante o processo costuma estar associado a um melhor prognóstico (Jung et al., 2015).

Em um processo terapêutico, espera-se que progressivamente o paciente possa ter novos modos de olhar a vida e o problema que o levou à terapia e, aos poucos, construir novos significados, visíveis por meio dos indicadores de mudança (Krause et al., 2006). É possível que os novos sentidos e perspectivas adquiridos traduzam-se em mudanças nas respostas adaptativas como frutos do trabalho realizado em conjunto com o terapeuta (Honda \& Yoshida, 2013). No caso estudado, a paciente teve a possibilidade de rever a percepção que tinha de si mesma, conforme sugerido por Krause et al. (2006). É possível que esse êxito decorra do fato de a paciente, conforme foi passando a fornecer respostas mais adequadas, pôde reformular a visão de si mesma, aumentando, consequentemente, a probabilidade do padrão de resposta mais adaptativa se generalizar para outras situações, inclusive às relacionadas ao setor Pr.

No presente estudo, se por um lado respostas mais adequadas parecem ter propiciado mudanças na vida da participante, por outro essas melhoras puderam ser captadas pelos IGM. Salienta-se ainda que estes podem oferecer pistas ao terapeuta no que diz respeito à resposta do paciente às suas intervenções, bem como sua adequação, na medida em que vai progredindo na hierarquia dos indicadores. Esse dado é especialmente importante quando se considera que o processo terapêutico é constituído por uma série de fases, onde mudanças ocorridas no final do tratamento (indicadores de maior nível hierárquico) dependem de mudanças anteriores, no início da terapia (indicadores de menor nível hierárquico). Estas estão relacionadas, principalmente, com a possibilidade do paciente poder rever a imagem e o conceito que tem de si mesmo e das relações que estabelece com o entorno onde vive (Fernández et al., 2015).

Os resultados terapêuticos não dependem somente de episódios de mudança isolados, mas de uma sequência de fases que começa com um pedido de ajuda e se estende até o término do tratamento. Krause et al. (2014) indicam que o paciente pode iniciar a terapia a partir de qualquer ponto descrito nos indicadores e que a mudança não é necessariamente linear. Apesar disso, espera-se 
que ao longo do processo determinadas mudanças ocorram em cada uma dessas fases, de modo que o paciente possa responder de forma mais adequada às vicissitudes da vida. Especificamente em relação ao caso estudado, a presença dos diversos indicadores ao longo do tratamento forneceu indicação da evolução da paciente, que foi apresentando respostas hierarquicamente superiores. A única exceção foi o indicador de número 17 (reconhecimento da ajuda recebida) na sessão cinco. Este é o único indicador de nível hierárquico elevado que pode aparecer em sessões iniciais ou mediais. Isso ocorre porque, muitas vezes, o paciente percebe mudanças em dificuldades pontuais, que começam a fazer diferença em sua vida.

Nesta pesquisa, a eficácia adaptativa e os indicadores presentes nos IGM foram analisados em uma tentativa de oferecer ao clínico uma visão mais abrangente do processo terapêutico. O distanciamento existente entre pesquisa e prática clínica pode ser abreviado ao se pensar nos IGM como um diferencial em relação a outros instrumentos, uma vez que sinalizam mudanças de forma hierárquica, contribuindo para oferecer indícios da evolução do paciente. Usados em conjunto, a avaliação da eficácia adaptativa e os IGM parecem permitir que terapeutas experientes e inexperientes identifiquem importantes aspectos do processo, seja na compreensão dos fatores que podem ensejar ou limitar a mudança, seja na avaliação de progresso do paciente submetido à psicoterapia.

Importante pontuar que esta pesquisa contou apenas com a análise de uma paciente submetida à psicoterapia considerada bem-sucedida. Sugere-se, portanto, a realização de novos estudos os quais compreendam maior número de casos, processos considerados mal sucedidos e, inclusive, com tratamentos em que houve abandono por parte do paciente.

\section{Colaboradores}

G.C. HONDA contribuiu para concepção e desenho do estudo, análise e interpretação dos dados, discussão dos resultados, revisão, e aprovação final do artigo. E.M.P. YOSHIDA contribuiu para concepção e desenho do estudo, análise e interpretação dos dados, discussão dos resultados e aprovação final do artigo. M. KRAUSE contribuiu para análise dos dados, discussão dos resultados, e revisão e aprovação final do artigo. G. de la PARRA contribuiu para análise dos dados e discussão dos resultados, revisão e aprovação final do artigo.

\section{Referências}

Altimir, C., Krause, M., de la Parra, G., Dagnino, P., Tomicic, A., Valdés, N., ... Vilches, O. (2010). Clients', therapists', and observers' agreement on the amount, temporal location, and content of psychotherapeutic change and its relation to outcome. Psychotherapy Research, 20(4), 472-487. https://doi. org/10.1080/10503301003705871

Arístegui, R., Reyes, L., Tomicic, A., Vilches, O., Krause, M., de la Parra, G., ... Valdés, N. (2004). Actos de habla en la conversación terapéutica. Terapia Psicológica, 22(2), 131-143.

Barber, J. P., Zilcha-Mano, S., Gallop, R., Barrett, M., McCarthy K. S., \& Dinger, U. (2014). The associations among improvement and alliance expectations, alliance during treatment, and treatment outcome for major depressive disorder. Psychotherapy Research, 24(3), 257-268. https://doi.org/10.1080/10503307. 2013.871080

Constantino, M. J., Vîslă, A., Ogrodniczuk, J. S., Coyne, A. E., \& Söchting, I. (2016). Looking forward: Correlates of posttreatment outcome expectation among depressed outpatients. Estudios de Psicología, 37(23), 282-310. https://doi.org/10.1080/02109395.201 6.1189205

Eells, T. D. (2007). Generating and generalizing knowledge about psychotherapy from pragmatic case studies. Pragmatic Case Studies in Psychotherapy, 3(1), 35-54. https://doi.org/10.14713/pcsp.v3i1.893

Fernández, O., Pérez, C., Gloger, S., \& Krause, M. (2015). Importancia de los cambios iniciales en la psicoterapia con adolescentes. Terapia Psicológia, 33(3), 247-255. https://doi.org/10.4067/S0718-48082015000300008

Honda, G. C., \& Yoshida, E. M. P. (2013). Mudança em psicoterapia: indicadores genéricos e eficácia adaptativa. Estudos de Psicologia (Natal), 18(4), 589-597. https://doi.org/10.1590/s1413$294 \times 2013000400006$ 
Jung, S. I., Serralta, F. B., Nunes, M. L. T., \& Eizirik, C. L. (2015). Desistência e conclusão em psicoterapia psicanalítica, um estudo qualitativo de pacientes em Porto Alegre, Brasil. Revista Brasileira de Psicoterapia, $17(1), 25-40$.

Krause, M., \& Altimir, C. (2016). Introduction: Current developments in psychotherapy process research. Estúdios de Psicologia, 37(2-3), 201-225. https://doi. org/10.1080/02109395.2016.1227574

Krause, M., Altimir, C., Pérez, J. C., \& de la Parra, G. (2014). Generic change indicators in therapeutic process with different outcomes. Psychotherapy Research, 25(5), 533-545. https://doi.org/10.1080/1 0503307.2014 .935516

Krause, M., de la Parra, G., Arístegui, R., Dagnino, P., Tomicic, A., Valdés, N., ... Ben-Dov, P. (2007). The evolution of therapeutic change studied through generic change indicators. Psychotherapy Research, 17(6), 673-689. https://doi.org/10.1080/10503300601158814

Krause, M., de la Parra, G., Arístegui, R., Dagnino, P., Tomicic, A., Valdés, N., ... Ramirez, I. (2006). Indicadores genéricos de cambio en el proceso psicoterapéutico. Revista Latinoamericana de Psicologia, 38(2), 299-325.
Simon, R. (1997). Proposta de redefinição da EDAO (Escala Diagnóstica Adaptativa Operacionalizada). Boletim de Psicologia, 47(107), 85-94.

Wampold, B. E. (2015). How important are the common factors in psychotherapy? An update. World Psychiatry, 14(3), 270-277. https://doi.org/10.1002/wps.20238

Wiseman, H., \& Tishby, O. (2014). The therapeutic relationship: Multiple lenses and innovations introduction to the special section. Psychotherapy Research, 24(3), 251-256. https://doi.org/10.1080/1 0503307.2014 .892648

Yoshida, E. M. P. (1999). EDAO-R: Precisão e validade. Mudanças (São Bernardo do Campo), 7(11), 189-213.

Yoshida, E. M. P. (2008). Mudança em psicoterapias breves: características de personalidade, conflito interpessoal e estratégia terapêutica (relatório de pesquisa). Manuscrito não publicado. Campinas: Pontifícia Universidade Católica de Campinas.

Recebido: setembro 22, 2015

Versão final: fevereiro 18, 2016

Aprovado: abril 15, 2016 